\title{
SUPERVISED MACHINE LEARNING BASED DYNAMIC ESTIMATION OF BULK SOIL MOISTURE USING COSMIC RAY SENSOR
}

\author{
Ritaban Dutta ${ }^{1}$, Claire D'Este ${ }^{2}$ \\ ${ }^{1}$ Research Scientist, ${ }^{2}$ Post Doctoral Fellow, Computational Informatics, CSIRO, Hobart 7001, Australia, \\ Ritaban.Dutta@csiro.au,claire.deste@csiro.au
}

\begin{abstract}
In this paper artificial neural network based sensor informatics architecture has been investigated; including proposed continuous daily estimation of area wise surface soil moisture using cosmic ray sensor's neutron count time series. Study was conducted based on cosmic ray data available from two Australian locations. The main focus of this study was to develop a data driven approach to convert neutron counts into area wise ground surface soil moisture estimates. Independent surface soil moisture data from the Australian Water Availability Project (AWAP) was used as ground truth. A comparative study using five different types of neural networks, namely, Feed Forward Back Propagation (FFBPN), Multi-Layer Perceptron (MLPN), Radial Basis Function (RBFN), Elman (EN), and Probabilistic networks (PNN) was conducted to evaluate the overall soil moisture estimation accuracy. Best performance from the Elman network outperformed all other neural networks with $94 \%$ accuracy with $92 \%$ sensitivity and $97 \%$ specificity based on Tullochgorum data. Overall high accuracy proved the effectiveness of the Elman neural network to estimate surface soil moisture continuously using cosmic ray sensors.
\end{abstract}

Index Terms: Artificial Neural Network, Surface Soil Moisture, Cosmic Ray Sensors, Neutron Counts.

\section{INTRODUCTION}

The Australian Cosmic Ray Sensor Soil Moisture Monitoring Network (CosmOz) [1-5] (Fig.1) is a near-real time continental scale soil moisture monitoring system originally inspired by the United States Cosmic-ray Soil Moisture Observing System (COSMOS) [3]. CosmOz aims to test the utility of the Hydroinnova CRS-1000 cosmic ray soil moisture probes [4] (Fig.1) for water management, water information, hydrological process research applications and test the feasibility and utility of a national near-real time soil moisture measurement network. The cosmic ray soil moisture probe measures the neutrons released when cosmic rays interact with hydrogen atoms in water molecules found in the soil and atmosphere. The number of fast neutrons emitted into the atmosphere is inversely correlated with soil moisture [1, 3]. In order to convert the neutron counts into surface soil moisture the currently available field calibration method for the cosmic ray sensors is very time consuming and inefficient. The method only considers two separate field calibrations to represent the whole year's soil moisture variation. The main focus of this study investigates the possibility of an alternative supervised data driven approach based on various artificial neural networks (ANN) to estimate surface soil moisture remotely and continuously. A comparative study using five different types of neural ANNs were conducted to evaluate the overall soil moisture estimation accuracy; Feed Forward Back Propagation, Multi-Layer Perceptron, Radial Basis Function,
Elman, and Probabilistic networks. Study was conducted based on cosmic ray data available from two Australian locations; Tullochgorum (Longitude $=147.9$, Latitude $=-41.7$ ) and Norwin (Longitude $=151.3$, Latitude $=-27.6$ ). Experimental time period for Tullochgorum was 2010/12/15 2013/01/02 (723 days), and 2011/05/11- 2013/01/03 (597 days) for Norwin [1-10].

\section{EXPERIMENTAL DESIGN}

\subsection{Cosmic Ray Sensor Data}

The Cosmic Ray sensor's most important data attributes are the atmospheric pressure corrected neutron count and rainfall, which have been used for this study as training and testing inputs. Fig. 2 shows the neutron count data recorded from the Tullochgorum location [1-20].

\subsection{Australian Water Availability Project (AWAP) Data}

The AWAP database is developed to monitor the state and trend of the terrestrial water balance of the Australian continent, using model-data fusion methods to combine both measurements and modeling. Time series data was extracted from the gridded map data based on latitude and longitude. Soil moisture data available from the AWAP has been used as 

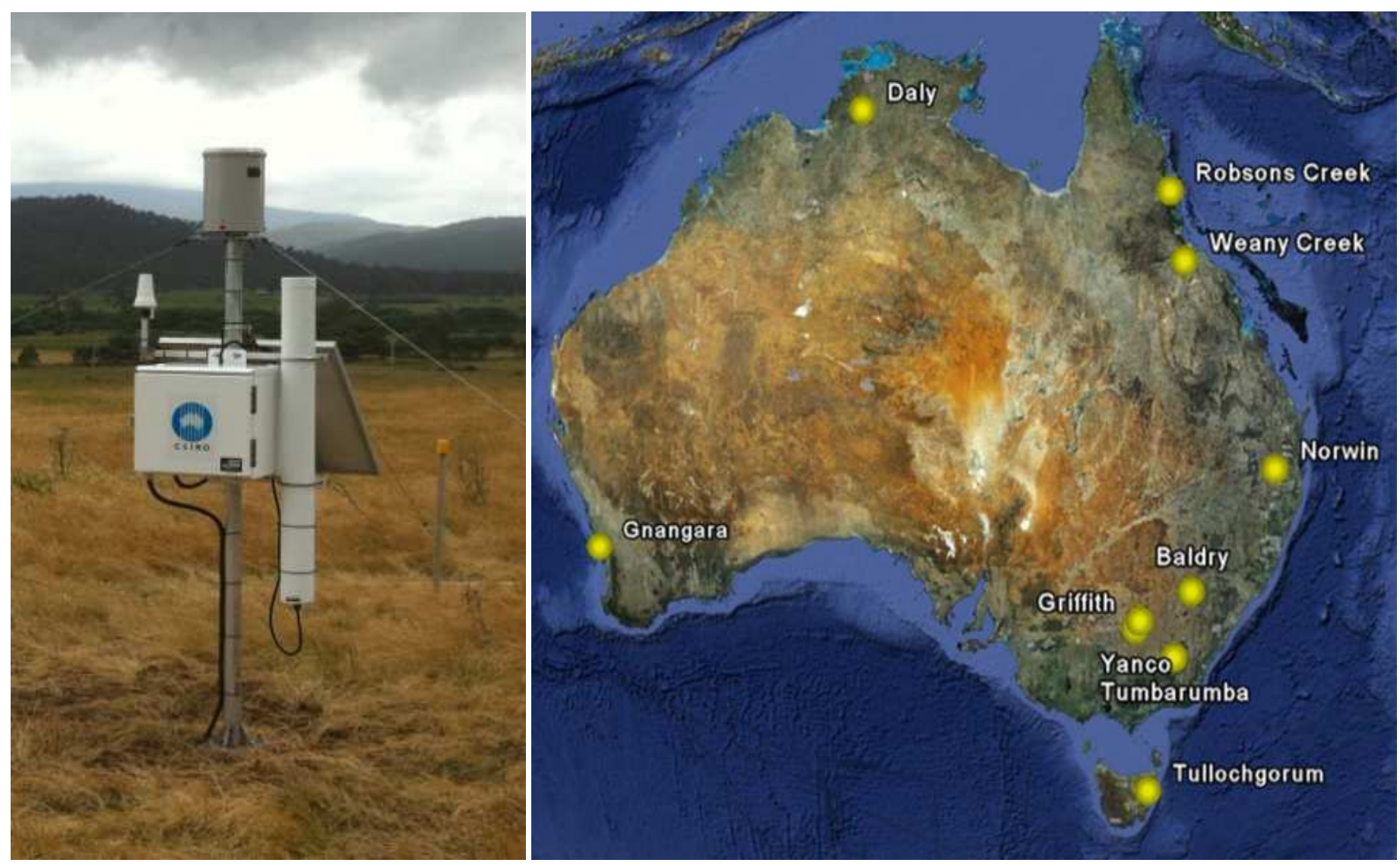

Fig -1: The Australian Cosmic Ray Sensor Network (CosmOz) and Hydroinnova CRS-1000 cosmic ray soil moisture probe deployed in Tullochgorum site.

external ground truth data to establish the cross correlation between recorded neutron counts and volumetric surface soil moisture. Available evapotranspiration data from AWAP has been used as an additional training and testing input to the ANNs (See Fig.2) [1-5].

\subsection{Problem Formulation}

Daily data for each of the inputs and target was recorded for both the locations. An incremental optimization algorithm was used to identify the best possible training and testing relative percentages where prediction accuracy was maximum with highest sensitivity and specificity. In the training phase daily average neutron counts, rainfall and evapotranspiration from the training input set were used to train ANNs against the corresponding AWAP daily soil moisture training target. Later in the testing phase, trained ANNs were simulated and tested using testing input set (unknown to the ANN paradigms) to produce estimated soil moisture. Estimated soil moisture profile from the testing phase was then evaluated against the testing target soil moisture profile from the AWAP, to establish the estimation performance accuracies [21-30].

\subsection{Methodology and Evaluation}

ANN requires input(s) and a known target for training the system. The training phase modulates the internal layers of the system based on the training inputs. Once the ANN system is trained, it is ready for testing. In the testing phase an ANN produces an output based on the combination of inputs. The Feed Forward Back Propagation, Multi-Layer Perceptron, Radial Basis Function, Elman, and Probabilistic networks were applied to the same data sets to establish the best architecture as indicated by prediction accuracy ((TP + $\mathrm{TN}) /(\mathrm{TP}+\mathrm{FN}+\mathrm{FP}+\mathrm{TN})$ where true positives $=\mathrm{TP}$, true negatives $=\mathrm{TN}$, false positives $=\mathrm{FP}$, false negatives $=\mathrm{FN})$. The evaluation process also included sensitivity (TP / (TP + $\mathrm{FN})$ ); specificity $(\mathrm{TN} /(\mathrm{FP}+\mathrm{TN})$ calculations to justify the estimation correctness [1-20]. 


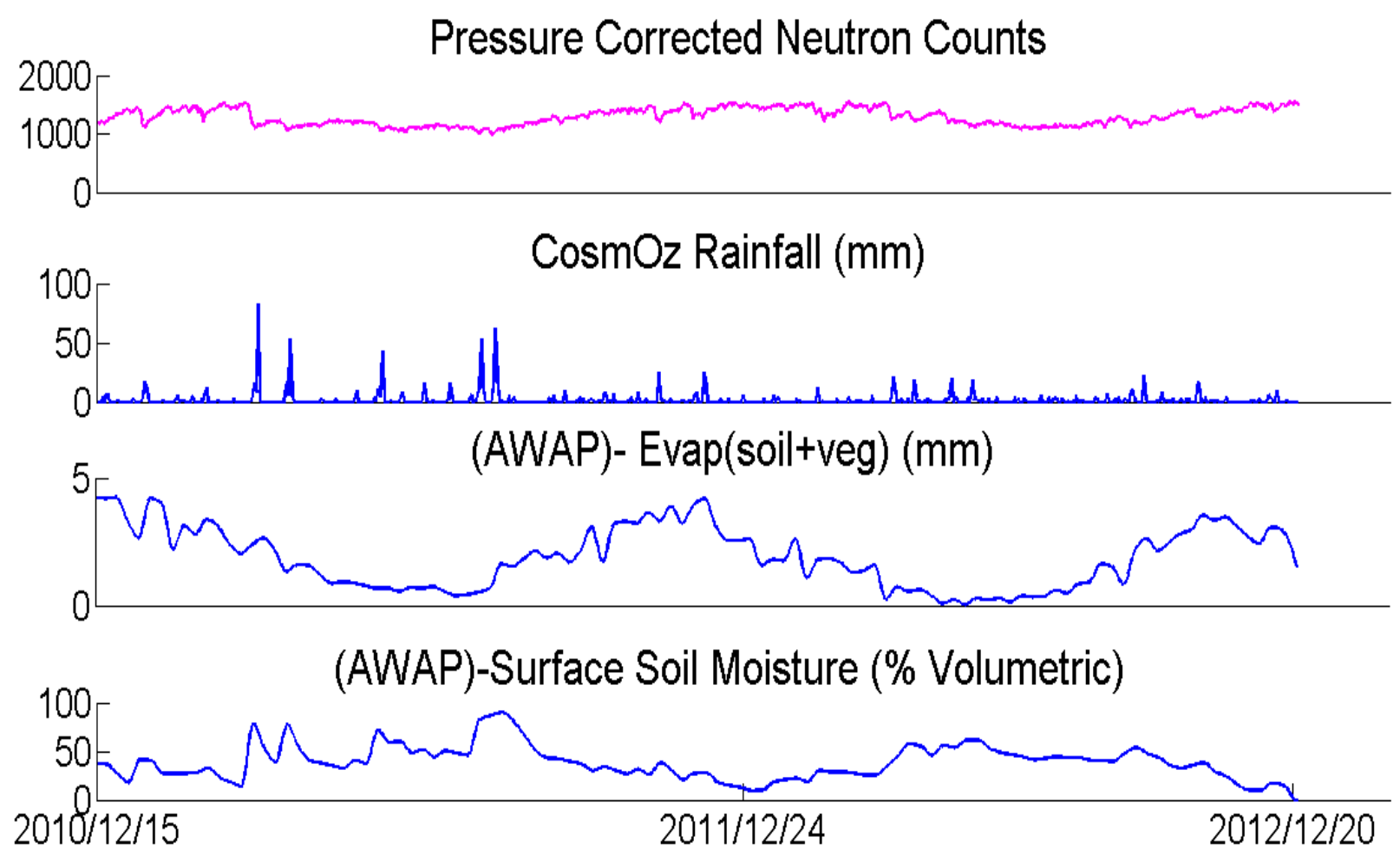

Fig -2: The CosmOz and AWAP data representing the 2010/12/15 - 2013/01/02 time period at Tullochgorum site, Tasmania.

\section{ESTIMATION RESULT}

A comparative study based on five different ANNs was conducted to identify the best network architecture with maximum generalization capability for estimating accurate surface soil moisture from unknown cosmic neutron counts. Based on Tullochgorum data it was found that maximum prediction accuracy from the

Elman network was achieved with $85 \%-15 \%$ training-testing paradigm, whereas for Norwin, it was $75 \%-25 \%$. Performance accuracies from the Tullochgorum data using FFBPN, MLPN, RBFN and PNN were $74 \%, 69 \%, 82 \%$ and $89 \%$ respectively; for Norwin data FFBPN, MLPN, RBFN and PNN were able to achieve $70 \%, 79 \%, 85 \%$ and $79 \%$ respectively.

The EN outperformed all other neural networks with $94 \%$ accuracy with $92 \%$ sensitivity and $97 \%$ specificity for Tullochgorum and with $91 \%$ accuracy with $95 \%$ sensitivity and $92 \%$ specificity for Norwin. Representative EN performances are depicted in Fig. 3. This study concluded that artificial neural network could be an effective alternative method for remote and continuous estimation of surface soil moisture using the recorded fast neutron counts using cosmic ray sensors [20-31].

\section{CONCLUSIONS}

This study concluded the Elman Neural network (ENN) could be an effective alternative calibration method for remote estimation of bulk soil moisture using the cosmic ray sensors. Better performance from the ENN could be explained physically. It is a simple recurrent neural network consisting of an input layer, a hidden layer, and an output layer. In this way it resembles a three layer feedforward neural network. ENN are very useful for predicting time series sequences, since they have a limited short-term memory. Short-term memory provides a unique capability to the ENN, by storing the previous learning step which could be used to influence the next learning step. At each time step, the input is propagated in a standard feed-forward fashion, and then a learning rule is applied. The fixed back connections result in the context units always maintaining a copy of the previous values of the hidden units (since they propagate over the connections before the learning rule is applied). Thus the network can maintain a sort of state, allowing it to perform such tasks as sequence-prediction that is beyond the power of a standard multilayer perceptron.

This study also concluded the supervised machine learning algorithms could be an effective alternative calibration method for remote estimation of bulk soil moisture using the cosmic ray sensors. Using AWAP database it was possible to train the 
ensemble estimators with long historical ground truth soil moisture data, which provided better generalization capability to predict accurate soil moisture from the cosmic neutron counts. Prediction results were very encouraging. Potentially this could help us to develop a remote virtual sensor calibration mechanism. This way cosmic ray sensor could be monitored and calibrated dynamically and virtually.

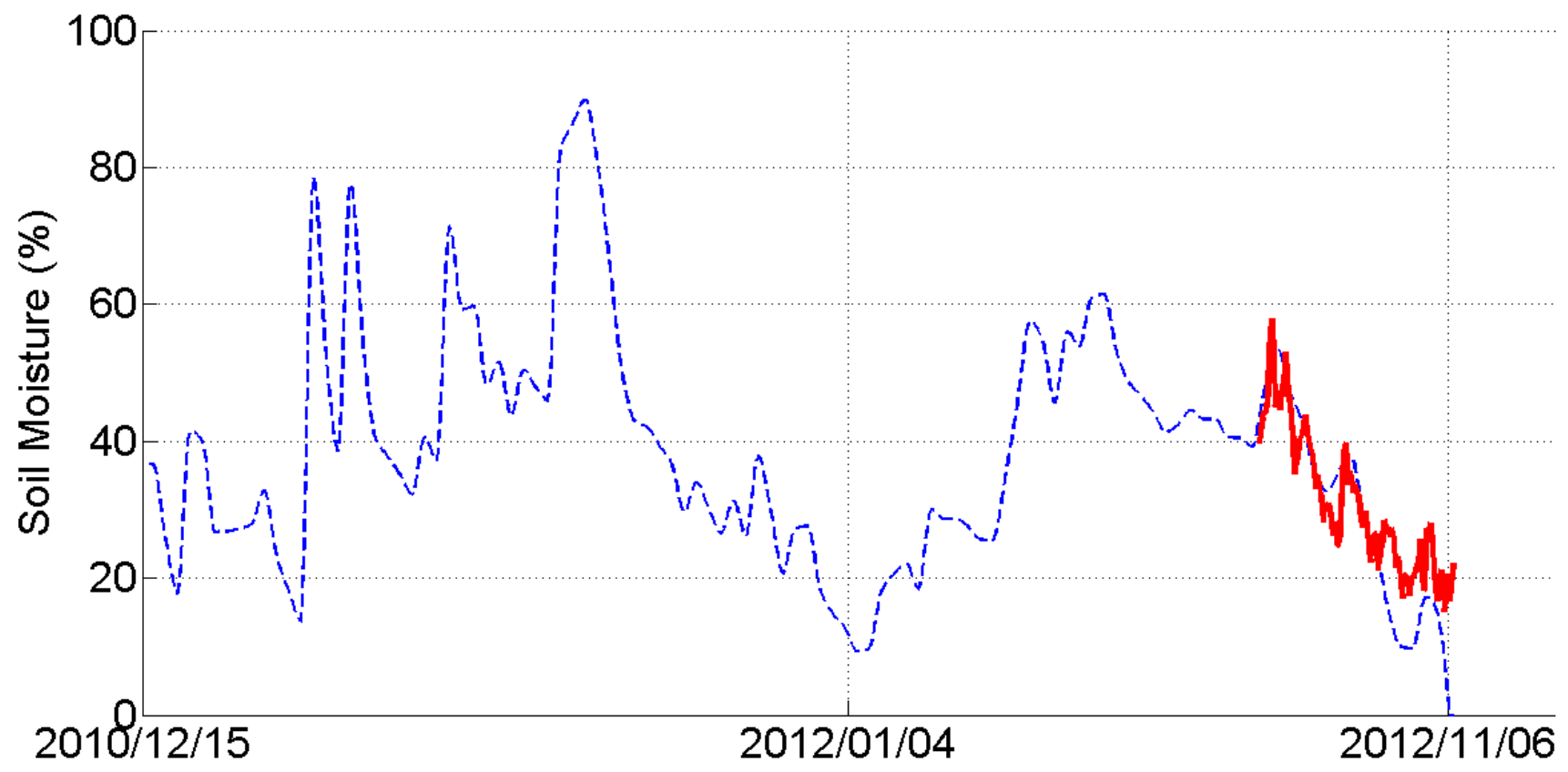

(a)

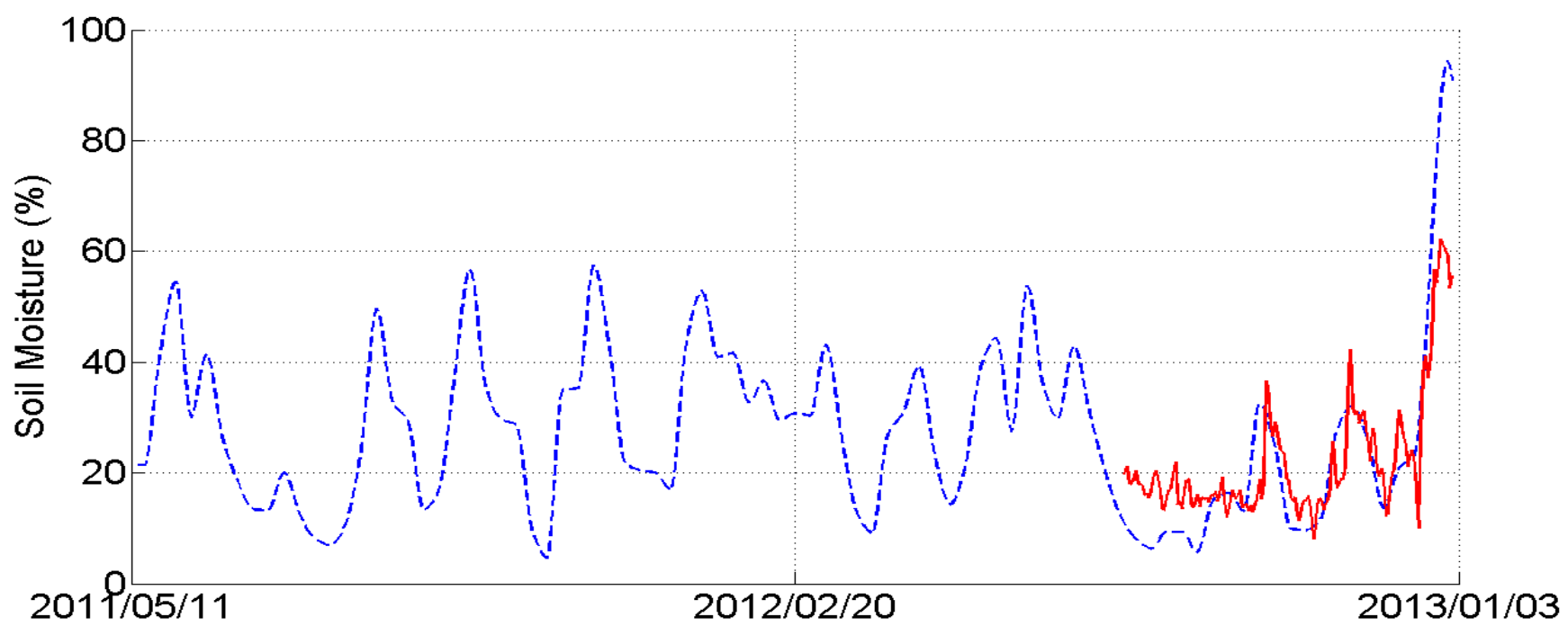

(b)

Fig -3: Elman Network based soil moisture estimation (red curves while blue represents testing target ground truth) during testing on (a) Tullochgorum and (b) Norwin data. 


\section{ACKNOWLEDGEMENTS}

The authors wish to thank the Intelligent Sensing and Systems Laboratory and the Tasmanian node of the Australian Centre for Broadband Innovation. A grant from the Tasmanian Government which is administered by the Tasmanian Department of Economic Development, Tourism and the Arts helped to conduct this research. The cosmic ray sensor data was made available through the $\mathrm{CosmOz}$ Australian soil moisture monitoring network funded by CSIRO and deployed by Dave McJannet, Aaron Hawdon and Rex Keen of CSIRO Land and Water. Soil samples for sensor calibration were collected by Bill Cotching of Tasmanian Institute of Agriculture. Data were delivered through CSIRO data service system developed by Peter Taylor. Authors would like to thanks Peter Briggs for providing access to the AWAP database. Authors would like to thanks Andrew Terhorst for his support of this project.

\section{REFERENCES}

[1] R. Dutta, A. Terhorst, A. Hawdon, B. Cotching, Bulk Soil Moisture Estimation Using CosmOz Cosmic Ray Sensor and ANFIS, IEEE Sensors 2012 Proceedings, Taipei, Taiwan, 9781-4577-1767-3/12, (2012), 741 -744.

[2] R Dutta, A Terhorst, "Adaptive Neuro-Fuzzy Inference System Based Remote Bulk Soil Moisture Estimation: Using CosmOz Cosmic Ray Sensor", IEEE Sensors Journal, Volume 13, Issue 6, pp. $2374-2381,2013$.

[3] http://hydroinnova.com/main.html Accessed July 2013.

[4] T.E. Franz, M. Zreda, R. Rosolem, T.P.A Ferre, A universal calibration function for determination of soil moisture with cosmic-ray neutrons. Hydrology and Earth System Sciences 17, (2013), 453-460.

[5] B. Hornbuckle, S. Irvin, T. E. Franz, R. Rosolem, and C. Zweck, The potential of the COSMOS network to be a source of new soil moisture information for SMOS and SMAP, paper presented at Proc. IEEE Intl. Geosci. Remote Sens. Symp., Munich, Germany, 2012.

[6] T. E. Franz, M. Zreda, T.P.A Ferre, R Rosolem, C. Zweck, S. Stillman, X. Zeng, W. J. Shuttleworth, Measurement depth of the cosmic ray soil moisture probe affected by hydrogen from various sources. Water Resources Research 48, (2012). [7] C.A. Rivera Villarreyes, G. Baroni, S. E. Oswald, Integral quantification of seasonal soil moisture changes in farmland by cosmic-ray neutrons. Hydrology and Earth System Sciences 15, (2011), 3843-3859.

[8] P. Carlson, A century of cosmic rays. Physics Today, 65, (2012), 30-36.

[9] D. Desilets, M. Zreda, Nature's neutron probe: landsurface hydrology at an elusive scale with cosmic rays. Water Resour. Res., 46, (2010), W11505, DOI: 10.1029 /2009WR008726.

[10] D. Desilets, M. Zreda, Spatial and temporal distribution of secondary cosmic-ray nucleon intensities and applications to in-situ cosmogenic dating. Earth Planet. Sc. Lett., 206, (2003), 21-42.
[11] E. Fermi, Artifical radioactivity produced by neutron bombardment, Nobel Prize lecture, (1938), 414-421.

[12] http://www.eoc.csiro.au/awap/ AWAP Website Accessed March 2013.

[13] R. R. Brooks, S. S. Iyengar, Multi-Sensor Fusion: Fundamentals and Applications with Software. Prentice Hall PTR, Upper Saddle River, New Jersey 07458, ISBN 0-13901653-8

[14] S. B. Kotsiantis, I. D. Zaharakis, and P. E. Pintelas. Supervised machine learning: A review of classification techniques, Frontiers in Artificial Intelligence and Applications 160 (2007): 3.

[15] T. Dietterich, Ensemble methods in machine learning, Multiple classifier systems (2000): 1-15.

[16] T. G. Dietterich, Approximate statistical tests for comparing supervised classification learning algorithms, Neural computation 10.7 (1998): 1895-1923.

[17] www.mathworks.com Accessed July 2013.

[18] J.-S.R. Jang, ANFIS: adaptive-network-based fuzzy inference system. IEEE Transactions on Systems, Man and Cybernetics, Volume: 23 , Issue: 3, (1993), $665-685$.

[19] P. P. V. D Smagt. "Minimisation methods for training feed-forward networks." Neural Networks 7.1 (1994): 1-11.

[20] S. Singhal, and W. Lance, Training feed-forward networks with the extended Kalman algorithm, IEEE International Conference on Acoustics, Speech, and Signal Processing, ICASSP-89, (1989).

[21] J. J. Hopfield, Learning algorithms and probability distributions in feed-forward and feed-back networks, Proceedings of the National Academy of Sciences 84.23, (1987), 8429-8433.

[22] J. L. Elman, Learning and development in neural networks: The importance of starting small, Cognition 48.1 (1993): 71-99.

[23] X. Z. Gao, X. M. Gao, and S. J. Ovaska, A modified Elman neural network model with application to dynamical systems identification, IEEE International Conference on Systems, Man, and Cybernetics, Vol. 2. (1996).

[24] S. C. Ahalt et al, Competitive learning algorithms for vector quantization, Neural networks 3.3 (1990): 277-290.

[25] P. Schneider, M. Biehl, and B. Hammer, Adaptive relevance matrices in learning vector quantization, Neural Computation 21.12 (2009): 3532-3561.

[26] R. Dutta, J.W. Gardner, E.L. Hines, "Classification of ear, nose, and throat bacteria using a neural-network-based electronic nose", MRS bulletin 29 (10), 709-713.

[27] A. Das, N.G. Stocks, A. Nikitin, E.L. Hines, "Quantifying stochastic resonance in a single threshold detector for random aperiodic signals", Fluctuation and Noise Letters 4 (02), L247L265, 2004.

[28] L. Hansen, P. Salamon, "Neural Network Ensembles," IEEE Trans.in Pattern Analysis and Machine Intelligence, vol. 12, no. 10, pp. 993-1001, 1990.

[29] M. P. Perrone, L. N. Cooper, "When Networks Disagree: Ensemble Methods for Hybrid Neural Networks," in Neural 
Networks for Speech and Image Processing, R. J. Mammone, Ed. Chapman-Hall, 1993, pp.126-142.

[30] A. Krogh, J. Vedelsby, "Neural network ensembles, cross validation, and active learning," in Advances in Neural Information Processing Systems, G. Tesauro, D. Touretzky, and T. Leen, Eds., vol. 7. The MIT Press, 1995, pp. 231-238.

[31] A. Krogh, P. Sollich, "Statistical mechanics of ensemble learning," Physical Review E, vol. 55, no. 1, pp. 811-825, 1997.

\section{BIOGRAPHIES}

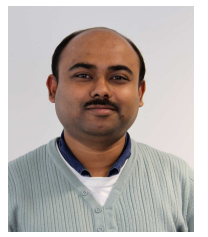

Dr. Ritaban Dutta is a Research Scientist in the field of Machine Learning within Intelligent Sensing and Systems Laboratory, CSIRO. Since 1999 he is undertaking research and development in all aspects of Machine Learning, Data Mining, Electronic Nose, and Knowledge Representation. In 2004 he was awarded PhD in engineering from University of Warwick, one of UK's elite universities. Ritaban has published over 80 peer-reviewed scientific research articles. He regularly acts as editor and reviewer for several international journals including IEEE and Elsevier Science.

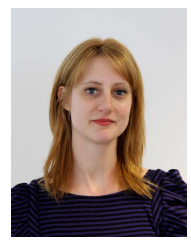

Dr Claire D'Este gained her $\mathrm{PhD}$ from the University of New South Wales and Sony Computer Science Laboratory, Paris. Her research has focused on incremental machine learning and human-robot interaction. At the Sony Computer Science Laboratory, she also worked on life-long learning methods for Sony's AIBO and Qrio robots. She has worked in Linux administration and support and was a senior developer in London creating telephone-based systems for media clients such as MTV and "The Sun" newspaper. 